\author{
Jean-Michel Jeannin
}

\section{Einleitung}

Spontan verbindet man mit dem Begriff «herzwirksame Glykoside» die Digitalis-Glykoside. In Tat und Wahrheit kennt man über 200 verschiedene herzwirksame Glykoside, die ausser in Digitalis-Arten in einer ganzen Reihe von weiteren Pflanzenarten vorkommen, z.B. Adonis vernalis (Frühlings-Adonisröschen), Convallaria majalis (Maiglöckchen), Nerium oleander (Oleanderstrauch), Strophanthus-Arten, Urginea (Scilla) maritima (Meerzwiebel) (Abb. 1). Nachfolgend werden die Chemie, Biochemie und Pharmakologie der einzelnen Substanzen besprochen und einige der Pflanzenarten, welche die Substanzen enthalten, exemplarisch in Wort und Bild vorgestellt. Die Toxikologie der vorgestellten Pflanzen wird jeweils bei der einzelnen Pflanze abgehandelt.

\section{Chemie und Biochemie der herzwirksamen Glykoside}

Die Grundstruktur der Glykoside besteht aus einem C17-Steroidgerüst mit einem ungesättigten 5- oder 6-gliedrigen Lactonring in 17-Stellung (Aglukon), an die Desoxyzucker glykosidisch gebunden sind. Glykoside mit einem 5-gliedrigen Lactonring heissen Cardenolide (Digitalis, Strophanthus, Convallaria), solche mit einem 6-gliedrigen Lactonring Bufadienolide (U. maritima). In Digitalis purpurea und D. lanata liegen die herzwirksamen Glykoside an Glukose bzw. an Glukose und Essigsäure gebunden vor ("genuine Glykoside»). Bei der Aufbereitung der Glykoside wird der Zucker durch natürliche Fermente abgespalten [1, 2]. Durch Abspalten des Zuckerrests wird aus einem

\title{
Phyto- und Mykotoxine (6)
}

Herzwirksame Glykoside

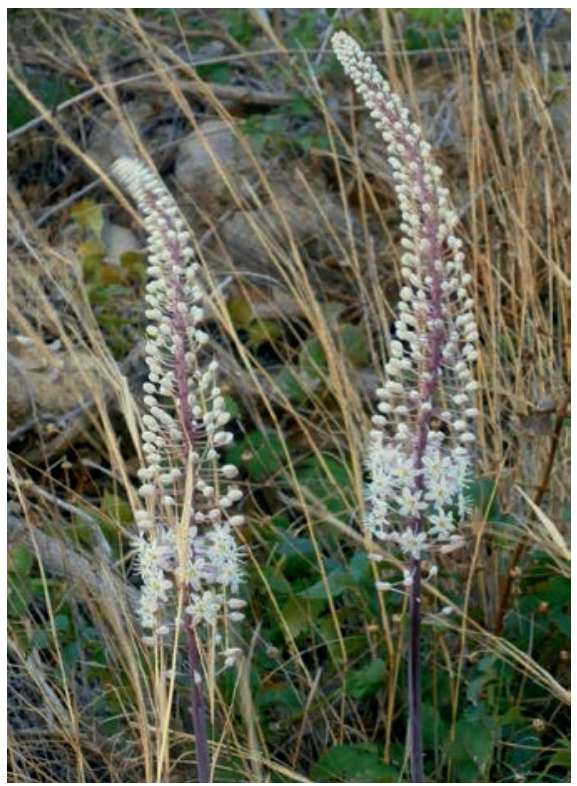

Abb. 1. Urginea (Scilla) maritima. Kreta, 2013.

Glykosid ein Genin. Die Herzwirksamkeit eines Glykosids wird entscheidend durch seine räumliche Konfiguration bestimmt. Die Art des Zuckers bzw. der Zucker hat darauf keinen Einfluss, weil Genine ebenfalls herzwirksam sind [2]. Hingegen hat der Zuckerrest einen grossen Einfluss auf die Pharmakokinetik eines Glykosids [1]. Glykoside aus Digitalis- und aus Strophanthus-Arten werden therapeutisch zur Behandlung der Herzmuskelinsuffizienz und bestimmter Herzrhythmusstörungen verwendet. Traditionell werden $\mathrm{Zu}$ bereitungen aus den Samen verschiedener Strophanthus-Arten und weitere Arten, die Glykoside enthalten, in Teilen Afrikas und in Indonesien als Pfeilgifte verwendet. Die älteste dokumentierte therapeutische Verwendung einer Pflanze mit herzwirksamen Glykosiden ist die von U. (Scilla) maritima. Sie wird in einem Papyrus um 1500 v. Chr. im alten Ägypten bezeugt [1].

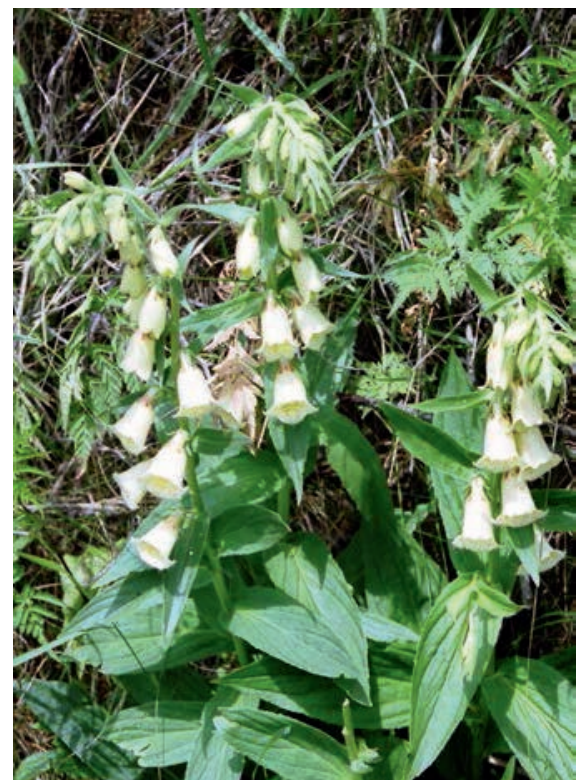

Abb. 2. Digitalis lutea. Engadin, 2006.

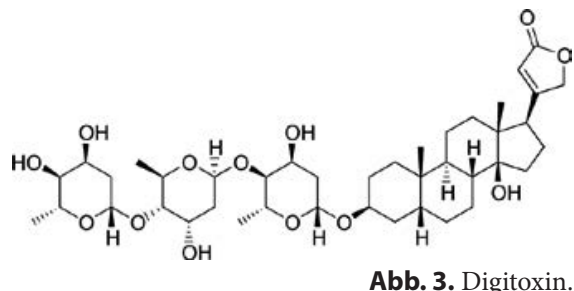

Abb. 3. Digitoxin.

\section{Digitalis ssp. (Plantaginaceae)}

Digitalis-Arten (Abb. 2) sind 2-jährige bis ausdauernde krautige Pflanzen aus der Familie der Plantaginaceae (Wegerichgewächse). Die Gattung ist in Asien und Europa heimisch, mit einem Schwerpunkt im südlichen Europa. Die 5-zähligen Blüten stehen in traubigen Ständen. Die Kronblätter sind röhrig bis glockenförmig verwachsen. Prominente Arten sind D. purpurea, D. lanata, D. lutea und D. grandiflora [3]. Die Inhaltsstoffe unterscheiden sich von Art zu Art: D. purpurea enthält bis zu 0,4\% Purpureaglykoside, Digitoxin (Abb. 3) und weitere Cardenolide, D. lanata bis zu 1,5\% Digoxin, Lantanosid, Gitoxin

\section{KARGER}

Fax +49761 4520714 (c) 2013 S. Karger GmbH, Freiburg
Dipl. med. biol. Jean-Michel Jeannin

Holeestrasse 43, 4054 Basel, Schweiz

jm.jeannin@medwiss.ch 


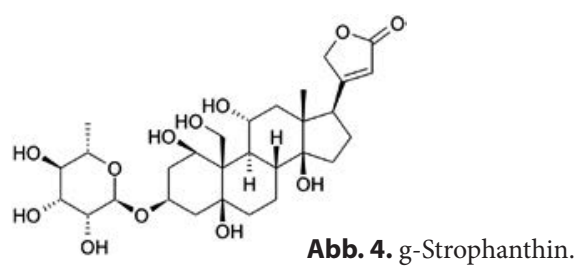

und verwandte Stoffe [4]. Therapeutisch eingesetzt werden hauptsächlich Digitoxin, Digoxin und Acetyldigoxin, eine halbsynthetische Substanz.

Digitalis gilt als hochgiftig (Klasse 1a). Etwa 0,3 g getrocknete Blätter sind bereits toxisch. Tödlich sind: 2,5-5 g für den Erwachsenen, $4-5 \mathrm{~g}$ für das Schwein, $5 \mathrm{~g}$ für den Hund, $150 \mathrm{~g}$ für das Rind und $25 \mathrm{~g}$ für das Pferd [4].

\section{Strophanthus ssp. (Apocynaceae)}

Strophanthus-Arten sind holzige Kletterpflanzen aus der Familie der Hundsgiftgewächse (Apocynaceae) mit einfachen glänzenden Laubblättern und trichterförmigen Blüten. Die Gattung ist im tropischen Afrika und im tropischen Asien heimisch. Strophanthus gratus enthält rund 30 herzwirksame Glykoside, darunter 3-8\% g-Strophanthin (Ouabain) (Abb. 4). $S$. kombé enthält bis zu $10 \%$ eines Gemischs mit k-Strophanthin und Cymarin. Aus S. speciosus wurde Christyosid isoliert. Therapeutisch eingesetzt werden g-Strophanthin und h-Strophanthin. Die Strophanthus-Glykoside gehören der Giftklasse 1a an, sind also äusserst giftig. Die Verabreichung von $1 \mathrm{mg}$ g-Strophanthin i.v. kann bereits tödlich sein [4].

\section{Urginea (Scilla) maritima (Asparagaceae)}

U. maritima ist eine ausdauernde krautige Pflanze aus der Familie der Spargelgewächse (Asparagaceae). U. maritima ist im Mittelmeerraum heimisch und stellt einen Artenkomplex dar [4-6]. Sie zeichnet sich durch eine kräftige grosse Zwiebel und durch grosse, breit lanzettliche Blätter in grundständiger Rosette aus, die im Frühsommer welken. Die kleinen, zahlreichen Blüten sitzen an einem bis zu 1,5 $\mathrm{m}$ hohen Blütenschaft [4]. Namentlich die Zwiebeln enthalten bis zu 1,8\% Bufadienolide und hierunter Glucoscillaren A, Scillaren A, die 11- $\beta$-hydroxylierten Derivate Scillaphäosid und Glucoscillaphäosid. Durch enzymatische Spaltung gewinnt man Proscillaridin A. Therapeutisch verwendet werden Proscillaridin A und Scillaren A. 1,5 g der Zwiebel sind tödlich [4].

\section{Pharmakologie der Digitalis- und Strophanthus-Glykoside}

Therapeutisch am wichtigsten sind Digitalis- und Strophanthus-Glykoside. Herzwirksame Glykoside steigern die Kontraktionskraft des Herzens (positive Inotropie), verringern die Frequenz (negative Chronotropie), mindern die Erregungsleitungsgeschwindigkeit (negative Dromotropie) und begünstigen durch eine Senkung der Reizschwelle die Erregungsbildung (positive Bathmotropie). Der Grundmechanismus der DigitalisWirkung besteht in einer Hemmung der membranständigen NatriumKalium-ATPase des Herzmuskels. Es bleibt vermehrt Natrium im Intrazellulärraum und in der Folge auch Kalzium. Eine Erhöhung der intrazellulären Kalziumkonzentration wiederum steigert die Kontraktilität des Herzmuskels. Insgesamt wird die Herzleistung verbessert, ohne dass der $\mathrm{O}_{2}$-Verbrauch ansteigt. Der Verbesserung der Herzleistung ist auch eine (erwünschte) Ausschwemmung kardial bedingter Ödeme zu verdanken. Wegen ihrer negativ dromotropen Wirkung eignen sich Digitalis-Glykoside ferner zur Behandlung von tachykarden Herzrhythmusstörungen, d.h. des Vorhofflimmerns. Die Wahl und wegen der geringen therapeutischen Breite auch die Dosierung eines Präparats müssen sehr individuell auf den einzelnen Patienten abgestimmt werden. Beispielsweise wird für das Erzielen eines raschen Wirkungseintritts g-Strophanthin empfohlen, wäh- rend Digitoxin für die meisten Fälle zu bevorzugen ist [2]. Die herzwirksamen Glykoside werden gleichmässig in alle Gewebe verteilt. Therapeutisch verwendet werden Digitoxin, Digoxin, Acetyldigoxin und g-Strophanthin. Digoxin wird in der Leber metabolisiert. Die Metaboliten sind gut wasserlöslich und werden teils mit der Galle ausgeschieden, teils diffundieren sie ins Gewebe zurück. Die mit der Galle ausgeschiedenen Metaboliten werden zu Digoxin umgewandelt und zusammen mit dem unveränderten Digoxin rückresorbiert. Digitoxin wird in einem komplexen Prozess metabolisiert und mit der Galle ausgeschieden, am Darm gespalten und rückresorbiert (enterohepatischer Kreislauf) [2].g-Strophanthin wird nach peroraler Verabreichung zu ca. $10 \%$ resorbiert [6] und unverändert über die Nieren ausgeschieden. Die Bedeutung der herzwirksamen Glykoside in der Therapie der Herzinsuffizienz ist zurückgegangen.

\section{Toxikologie der \\ herzwirksamen Glykoside}

Die sogenannte therapeutische Breite der herzwirksamen Glykoside ist sehr klein: Eine Überschreitung der voll wirksamen Dosis um das Eineinhalb- bis Dreifache ist bereits toxisch. Arrhythmien und Erbrechen treten bei einer Vergiftung als Erstes auf bei empfindlichen Patienten noch vor dem Erreichen der voll wirksamen Dosis. Schwere Vergiftungen münden in Kammertachykardie und tödliches Kammerflimmern [2].

\section{Literatur}

\footnotetext{
1 Reichstein T: Chemie der herzaktiven Glykoside. Angewandte Chemie 1951;63:412-421.

2 Kuschinsky G, Lüllman H: Kurzes Lehrbuch der Pharmakologie, ed 5. Stuttgart, Thieme, 1971.

3 Fingerhüte. http://de.wikipedia.org/wiki/ Fingerhüte.

4 Wink M, et al: Handbuch der giftigen und psychoaktiven Pflanzen. Stuttgart, WVG, 2008

5 Weisse Meerzwiebel. http://de.wikipedia.org/ wiki/Weiße_Meerzwiebel.

6 g-Strophanthin. http://de.wikipedia.org/wiki/
} G-Strophanthin. 